staging operation with extraperitoneal SLNB. First, each $1 \mathrm{ml}$ of indocyanine green (ICG) diluted at $1.25 \mathrm{mg} / \mathrm{ml}$ was injected into the submucosa and stroma at 3 and 9 o'clock of the cervix. Second, we opened the mid-vagina between the cervix and vaginal orifice where the pelvic floor muscles were palpated. Third, a space was secured by placing a finger upward and outward after ensuring the opening space of the mid-vagina. Thereafter, we inserted single port trocar through the opening, and then we found the para-cervix including the pelvic autonomic nerves, and the obturator nerve. After peeling off the pelvic vessels and nerves and ureter, we found the sentinel lymph node near the external iliac vessel, and resected it. Then, we performed vNOTES hysterectomy, and she was discharged on the second day of surgery without any complications.

Conclusions vNOTES staging operation with extraperitoneal SLNB may be feasible, and the determination over the extraperitoneal opening may be important to ensure the adequate view to identify the pelvic structure for patients with a nonprolapsed uterus and low-risk endometrial cancer.

\section{SF009/\#460 MINIMALLY INVASIVE SECONDARY CYTOREDUCTIVE SURGERY FOR CELIAC AND CARDIOPHRENIC ISOLATED NODAL RECURRENCE OF OVARIAN CANCER}

${ }^{1} V$ Gallotta, ${ }^{2} \mathrm{M}$ Loverro*, ${ }^{3} \mathrm{R}$ Ergasti, ${ }^{4} \mathrm{D}$ Nachira, ${ }^{1} \mathrm{C}$ Conte, ${ }^{2} \mathrm{~V}$ Gallitelli, ${ }^{5} \mathrm{G}$ Scaglione, ${ }^{3} \mathrm{~A}$ Fagotti, ${ }^{2} \mathrm{G}$ Scambia. ${ }^{1}$ Fondazione Policlinico Universitario Agostino Gemelli, IRCCS, Gynecologic Oncology, Rome, Italy; ${ }^{2}$ Università Cattolica del Sacro Cuore, Department of Woman and Child Health and Public Health, Woman Health Area, Fondazione Policlinico Universitario A. Gemelli Irccs, Roma, Italy; ${ }^{3}$ Fondazione Policlinico Universitario A. Gemelli, IRCCS, Department of Woman and Child Health and Public Health, Rome, Italy; ${ }^{4}$ Fondazione Policlinico Universitario A. Gemelli IRCCS, Roma, Italy; Università Cattolica del Sacro Cuore Sede di Roma, Roma, Italy, Department of Thoracic Surgery, Rome, Italy; ${ }^{5}$ Fondazione Policlinico Universitario A. Gemelli IRCCS, Roma, Italy, Gynecopathology and Breast Pathology Unit, Roma, Italy

\subsection{6/ijgc-2021-IGCS.53}

Introduction Despite the advances in cytoreductive efforts and frontline chemotherapy in advanced ovarian cancer (OC), recurrence is a common event, with $>70 \%$ of women experiencing relapse within two years of from diagnosis. The standard treatment for recurrent ovarian cancer (ROC) patients has been traditionally represented by systemic chemotherapy; however, this concept has been recently recognized as presenting a greater level of complexity given the influence of histotype, status of BRCA genes, previous antiangiogenetic treatment and pattern of relapse presentation. Several retrospective studies, as well as randomized prospective trials suggested that secondary cytoreductive surgery (SCS) could provide better oncological outcomes in platinum-sensitive ROC patients, in case of complete cytoreduction, which has to be considered the goal to be achieved.

Description As far as lymph node relapse is concerned, some biological and clinical lines of evidence suggest that lymph node recurrences from OC would be better managed with SCS than medical treatment alone, given a relatively more indolent clinical behaviour compared to parenchymal and peritoneal disease. However, the documentation of lymph nodes metastasis in the hepatoceliac and cardiophrenic region at the time of SCS might be considered as a challenging clinical and surgical scenario. Although surgical management by minimally invasive surgery (MIS) could be expected to represent a demanding task in SCS, this video provides a step-by-step description of the surgical technique adopted for hepatoceliac and cardiophrenic lymph nodes resection.

Conclusion MIS is feasible and could be a viable option for selected cases of ROC, minimizing the intra- and post- operative complications.

\section{SF010/\#77 LAPAROSCOPIC SENTINEL LYMPH NODE BIOPSY FOR STAGE I ENDOMETRIAL CANCER: DUAL TRACER METHOD}

M Slimane, M Ghalleb*, H Bouaziz, M Bouhani, R Chargui, K Rahal. Salah Azaiez Institute of Oncology, Surgical Oncology, Tunis, Tunisia

\subsection{6/ijgc-2021-IGCS.54}

Introduction Sentinel lymph node (SLN) biopsy is a new trending option in the management of stage I endometrial cancer. The first results are showing non inferiority when compared to the classic pelvic lymphadenectomy dissection and also a decrease of morbidity especially lymphedema. Our Aim is to Share one of our cases with the IGCS community.

Description We report the case of a 50 year old women with a stage Ia Endometrial cancer. She was included into our institution Trial to see the feasibility of SLN biopsy in endometrial cancer. The patients have been treated laparoscopically and the Dual tracer method was used to detect the SLN. A four trocars approach was used. A $10 \mathrm{~mm}$ trocars in the umbilicus and one in the left iliac fossa, Two five $\mathrm{mm}$ in the hypogastric region and right iliac fossa. After careful abdominal exploration we detected the blue dye in the left iliac region. We started with opening the left pelvic wall peritoneum. Careful dissection of a blue enlarged lymph node. After the extraction the blue lymph node was also found to have a high radioactivity. The frozen section was negative. As a part of our protocol the women had a full lymph-node dissection and no metastatic lymph-node was found.

Conclusion SLN biopsy in stage one endometrial cancer seems to be technically feasible. We are waiting for the result of our trial to implemented in our current standards of care.

\section{SF011/\#197 TRANSVAGINAL ENDOSCOPIC RESECTION (TVER) OF INVASIVE VAGINAL LESION}

${ }^{1} \mathrm{~N}$ Dogra*, ${ }^{2} \mathrm{R}$ Linder, ${ }^{2} \mathrm{~J}$ Murphy, ${ }^{2} \mathrm{~T}$ Zigras. ${ }^{1}$ University of Toronto, Obstetrics and Gynecology, Toronto, Canada; ${ }^{2}$ Trillium Health Partners, Credit Valley Hospital, Gynecology Oncology, Mississauga, Canada

\subsection{6/ijgc-2021-IGCS.55}

Objective This video demonstrates a novel surgical technique for excision of an invasive vaginal lesion using a single port laparoscopic access device.

Study Methods A 31 year old female with a history of SLE on azathioprine presented with recurrent HSIL of cervix. A LEEP showed microinvasion measuring $2 \mathrm{~mm}$ and a vaginal fornix biopsy showed HSIL, but was clinically suspicious for invasion. The patient underwent a transvaginal endoscopic resection (TVER) of the lesion for diagnostic purposes. A single port laparoscopic access device was used for colpopneumo-occlusion. The endoscope was inserted into the vagina, and the $1.5 \mathrm{~cm}$ lesion was resected with margins of 5 
$\mathrm{mm}$ under direct visualization using monopolar cautery. The mucosa edges were reapproximated with V-lock suture.

Results Pathology showed squamous mucosa with high-grade vaginal intraepithelial neoplasia and a focal area of invasion (with depth of invasion $<1 \mathrm{~mm}$ ). The patient tolerated the procedure well and was discharged from Day Surgery without any postoperative complications.

Conslusion Transvaginal endoscopic resection for superficially invasive vaginal lesions can be performed safely and provide accurate diagnosis with excellent visualization.

\section{SF012/\#204 10 STEPS TO APPROACH LARGE OVARIAN MASSES THROUGH VNOTES (VAGINAL NATURAL TRANSLUMINAL ENDOSCOPIC SURGERY)}

'L Badiglian-Filho*, ${ }^{1} \mathrm{G}$ Baiocchi, ${ }^{1} \mathrm{C}$ Chaves Faloppa, ${ }^{2}$ J Baekelandt. ${ }^{1}$ AC Camargo Cancer Center, Gynecologic Oncology, Sao Paulo, Brazil; '2Imelda Hospital, Gynecologic Oncology, Bonheiden, Belgium

\subsection{6/ijgc-2021-IGCS.56}

10 steps to approach large ovarian masses through vNOTES. Introduction VNOTES (vaginal Natural Orifices Transluminal Endoscopic Surgery) is a novel technique that allows a laparoscopic approach to the pelvic and abdominal cavity through the vagina. It seems to facilitate large ovarian masses (with benign characteristics) removal when compared to conventional laparoscopy.

Description We recommend 10 steps to approach large ovarian masses through vNOTES. Each step is explained in the surgical video.

Step 1. Select suitable case.

Step 2. Setting up the patient and the surgical team.

Step 3. Accessing the cavity.

Step 4. Inserting vaginal port.

Step 5. Cavity inspection.

Step 6. Sealing and division.

Step 7. Bag the cyst.

Step 8. Cavity revision.

Step 9. Revise the inner ring of the vaginal port.

Step 10. Closing the vaginal vault.

Conclusion It is feasible to approach large ovarian masses through vNOTES.

\section{SF013/\#236 ROBOTIC APPROACH FOR A TOTAL HYSTERECTOMY BILATERAL SALPINGO- OOPHORECTOMY AND SUCTION CURETTAGE OF A 20-WEEK SIZE UTERUS WITH GESTATIONAL TROPHOBLASTIC NEOPLASIA}

${ }^{1} V$ Gupta*, ${ }^{2} S$ Salvador, ${ }^{2}$ W Gotlieb, ${ }^{2} S$ Lau. 'McGill University, Gynaecologic Oncology, Montreal, Canada; ${ }^{2}$ McGill University, Jewish General Hospital, Gynecology Oncology, Montreal, Canada

\subsection{6/ijgc-2021-IGCS.57}

Introduction Gestational trophoblastic neoplasia (GTN) is a malignant trophoblastic disease following either molar or nonmolar pregnancies. GTN is primarily treated through uterine evacuation using suction curettage followed by observation or adjuvant chemotherapy based on WHO risk scoring. ${ }^{1,2}$ In patients who have completed child-bearing, hysterectomy is an acceptable option and may decrease time to remission and required chemotherapy cycles. ${ }^{2}$ In patients presenting with large volume uterine disease, evidence of metastasis, and highrisk WHO scoring, patients are treated with multi-agent chemotherapy including Etopisode, Methotrexate, Actinomycin-D, Cycophosphamide, and Vincristine (EMA-CA). ${ }^{1,2}$ EMA-CO has significant risks of complications, including acute hemorrhage and trophoblastic emboli. ${ }^{2,3}$ In patients with large uteri, surgical risks include uterine perforation and acute hemorrhage, ${ }^{4}$ requiring a large laparotomic incision. Accordingly, there is a need for risk-reducing minimally invasive approaches in the surgical treatment of GTN.

Description The patient is a 53 year old G4P4 presenting with an enlarged uterus of $20 \mathrm{~cm}$ with snowstorm appearance, a beta-hCG >400 000IU, lung metastases and a WHO risk score of 8 . Preoperatively her blood pressure was 168/105. She underwent a robotic total hysterectomy and bilateral salpingo-oophorectomy, and guided suction curettage. Blood loss was minimal. The patient was scored post-procedure as WHO low risk (3). She received methotrexate, and switched to Actinomycin-D after a plateau in beta-hCG. Her beta-hCG is normal 5 months later.

Conclusion We present a minimally invasive approach that ameliorates the surgical and chemotherapy risks of uterine rupture, acute hemorrhage, and trophoblastic emboli, with a normalization of beta-hCGs after treatment with single-agent chemotherapy.

\section{SF014/\#370 LAPAROSCOPIC REPAIR OF OBTURATOR NERVE INJURY AT THE TIME OF PELVIC LYMPHADENECTOMY}

R Linder*, J Gomez, T Feigenberg. Trillium Health Partners, Credit Valley Hospital, Gynecology Oncology, Mississauga, Canada

\subsection{6/ijgc-2021-IGCS.58}

Introduction The obturator nerve is formed by the lumbar plexus, receiving its fibers from the anterior division of L24. Clinically, obturator nerve injury manifests with sensorial loss at the medial aspect of thigh, pain at medial portion of the groin and ipsilateral adductor weakness. The risk of obturator nerve injury is increased during pelvic lymphadenectomy and surgeries for gynecologic malignancies. Here, we present a video film of full-thickness transection of obturator nerve at the time of pelvic lymphadenectomy in a patient who underwent laparoscopic surgery for endometrial cancer. The transected nerve was primarily repaired through a laparoscopic approach at the time of surgery. The patient experienced a full recovery with no motor function deficiencies.

Description In this surgical film we present an 83-year-old woman, G2P2, who was diagnosed with a grade 3 endometrioid endometrial adenocarcinoma. She underwent a laparoscopic hysterectomy and sentinel lymph-node biopsies as well as resection of enlarged external iliac node. During the procedure, we identified a complete transection of the obturator nerve. To enable an end to end repair, the external iliac artery and vein were mobilized. The proximal end of the nerve was identified lateral to these vessels. An end-to-end anastomosis of the nerve was performed using Prolene sutures. The patient experienced an uneventful post-operative recovery. She discharge home on post-operative day 2 , able to ambulate independently with no assistance. 